Pacific

Journal of

Mathematics

ELECTRICAL RESISTANCE OF $\boldsymbol{N}$-GASKET FRACTAL NETWORKS

Brighid Boyle, Kristin Cekala, David Ferrone, NeIL RIFKIN AND ALEXANDER TEPLyAeV 


\title{
ELECTRICAL RESISTANCE OF $N$-GASKET FRACTAL NETWORKS
}

\author{
Brighid Boyle, Kristin CEKala, DAVid Ferrone, \\ NeIl RifKin AND AleXANDER TePlyaEV
}

\begin{abstract}
We study self-similar local regular Dirichlet, or energy, forms on a class of fractal $N$-gaskets, which are generalizations of polygaskets. This is directly related to self-similar diffusions and resistor networks (electrical circuits). We prove existence and uniqueness, and also obtain explicit formulas for scaling factors and resistances (transition probabilities). We also study asymptotic behavior of these quantiles as the number of "sides" $N$ of an $N$ gasket tends to infinity.
\end{abstract}

\section{Introduction}

This paper is part of a relatively new, but now well-established, field of analysis and probability on fractals, see [Kigami 1993; 1994; 2001; Barlow 1998; Strichartz 1999a; 2006; Mosco 2002; Adams et al. 2003; Stanley et al. 2003; Meyers et al. 2004; Teplyaev 2004; $\geq 2007$; Bajorin et al. $\geq 2007$ ], and references therein for a sample of mathematical literature on the analysis on fractals. Furthermore, many of the questions addressed here are related to the general Dirichlet form theory; for further information on this subject the reader can refer to the now classical books [Bouleau and Hirsch 1991; Fukushima et al. 1994]. To be more precise, we are interested in local regular self-similar Dirichlet, or energy, forms on a subclass of finitely ramified self-similar fractals. Nonlocal Dirichlet forms on fractals have been also extensively studied, see for instance [Zähle 2005; Hansen and Zähle 2006] and references therein.

Roughly speaking, self-similar fractals are objects that can be divided, with infinite detail, into smaller objects of similar shape. A snowflake is a classic example. The properties and physical applications of various types of these objects have been the subject of many studies in physics, of which we mention only a few [Gefen et al. 1983; Alexander 1984; Rammal and Toulouse 1983; Rammal

MSC2000: primary 28A80; secondary 94C99, 60J45, 34B45, 31C25.

Keywords: self-similar Dirichlet form, energy form, fractal, polygasket, $N$-gasket, resistor network, electrical circuit.

Research supported in part by the NSF grant DMS-0505622. 
1984; Stinchcombe 1989]. Fractal antennas were studied and successfully tested, in particular, in wireless devices [Puente-Baliarda et al. 1998; Hohlfeld and Cohen 1999; Musser 1999].

One particular type of self-similar fractal objects, known as polygaskets, are formed by dividing a regular polygon into smaller regular polygons of the same shape, arranged in a circular pattern [Strichartz 1999a; 1999b; 2000; 2006; Adams et al. 2003; Tyson and Wu 2005; 2006]. If a polygasket is finitely ramified (that is, point connected), then it belongs to the class of so-called nested fractals [Lindstrøm 1990; Metz 1996; Strichartz 2006]. The $N$-gaskets belong to the larger class of so-called p.c.f. self-similar sets [Kigami 1993; 2001], and are generalizations of finitely ramified polygaskets.

In this study we consider self-similar fractal resistor networks (electrical circuits) or, equivalently, a self-similar energy (Dirichlet) form on an $\mathrm{N}$-gasket. Using a combination of various methods of mathematical analysis, some of which come from electric engineering (see the Appendix), we construct networks with the property that every resistor in a network of a given order is a scalar multiple of the corresponding resistor in the next-order network, this scalar being constant across the fractal. This allows to translate the resistance of these fractal electrical networks into conventional, easily manipulated networks. We prove existence and uniqueness, up to a multiplicative constant, of a self-similar resistance (Dirichlet, energy) form on any $N$-gasket. In general this is a difficult and delicate question [Metz 1993; 1996; 2003; Sabot 1997; Hambly et al. 2006; Peirone 2006]. We derive explicit formulas which uniquely determine all the resistances and scaling constant for every $\mathrm{N}$-gasket. Furthermore, we explore the behavior of these constants as certain parameters tend to infinity, thus capturing the general properties of the current flow in an $N$-gasket with large $N$.

This paper is organized as follows. In Section 1 , we define $N$-gaskets and state our main objectives. In Section 2, we transform the self-similar structure of an $N$-gasket to simplify the problem and make available some ideas from electrical engineering. In Section 3, we compute the resistance scaling factor $c$, which is one of our main results, and also the resistances in the transformed network. In Section 4, we use the results of the previous sections to compute the resistances in the original problem. In Section 5 we study various asymptotic behaviors of the resistance scaling factor $c$ and of resistances, as the parameters of an $N$-gasket approach infinity. In particular, we compute the asymptotic dimension in the effective resistance metric and the asymptotic spectral dimension in Corollary 5.8. In the Appendix, we describe briefly the relevant electrical circuits formalism, prove an auxiliary result used in the main body of the paper, relate our results to the theory of random walks, and give some related references. 


\section{Definitions and examples}

An $N$-gasket is a self-similar fractal $F$ which is a compact metric space made of $N$ scaled copies $F_{1}, \ldots, F_{N}$ of itself, that is,

$$
F=\bigcup_{j=1}^{N} F_{j} .
$$

These copies $F_{j}$ are called depth-1 cells, or 1-cells for short. The self-similarity means that there are contractive homeomorphisms

$$
\psi_{j}: F \rightarrow F_{j} \quad \text { for each } j=1, \ldots, N .
$$

By definition, the boundary of the $N$-gasket consists of $N$ points $v_{1}, \ldots, v_{N}$, one in each 1-cell, with the property $\psi_{j}\left(v_{j}\right)=v_{j}$. The boundary of the $k$-th scaled copy $F_{k}$ of an $N$-gasket consists of points $v_{k, 1}, \ldots, v_{k, N}$ numbered so that $v_{k, j}=\psi_{k}\left(v_{j}\right)$. In particular, $v_{k}=v_{k, k}$.

Notation 1.1. When numbering cells in an $N$-gasket we always use cyclic notation modulo $N$, that is $F_{N+1}=F_{1}, v_{N+1}=v_{1}$ etc.

Up to a homeomorphism, an $N$-gasket is defined by three numbers $N_{1}, N_{2}, N_{3}$ with $N_{1}+N_{2}+N_{3}=N$. The most important property of an $N$-gasket is that

$$
F_{k} \cap F_{k+1}=v_{k, k+N_{3}}, \quad F_{k} \cap F_{k-1}=v_{k, k+N-N_{2}},
$$

and the depth-1 cells are otherwise disjoint (note that we use cyclic notation as in Notation 1.1). Thus, we have identifications $v_{k, k+N_{3}}=v_{k+1, k+1+N-N_{2}}$. This means that each $k$-th scaled copy $F_{k}$ of the $N$-gasket is connected to the other scaled copies via its boundary points $v_{N_{3}+k}^{\prime}$ and $v_{N-N_{2}+k}^{\prime}$, if we denote $v_{k, j}=v_{j}^{\prime}$. Thus, we will sometimes call an $N$-gasket with these connectivity properties the $\left(N_{1}, N_{2}, N_{3}\right)$ gasket. In Figure 1, one can see that the Sierpiński gasket is a $(1,1,1)$-gasket, the pentagasket is a $(1,2,2)$-gasket, and the hexagasket is a $(2,2,2)$-gasket. The so-called fractal cut square and the fractal red cross in Figure 2 are respectively a $(2,1,1)$-gasket and a $(1,1,2)$-gasket.

From the general theory [Kigami 1993; Hveberg 2005], an $N$-gasket exists for any triple $\left(N_{1}, N_{2}, N_{3}\right)$ of positive integers with $N=N_{1}+N_{2}+N_{3}$. An $N$-gasket can be easily constructed as a factor space of the one-sided shift space $\{1, \ldots, N\}^{\mathbb{N}}$, as in [Kigami 1993, Appendix]. Moreover, an $N$-gasket is defined uniquely up to a homeomorphism by the triple $\left(N_{1}, N_{2}, N_{3}\right)$, except that an $\left(N_{1}, N_{2}, N_{3}\right)$-gasket and an $\left(N_{1}, N_{3}, N_{2}\right)$-gasket are homeomorphic via the obvious "reflection" map.

Note that any polygasket, and any nested fractal in general, has by definition a dihedral symmetry group. It is easy to see from the definition that for any $N$-gasket there is a homeomorphism $\rho$ such that $\rho \circ \psi_{j}=\psi_{j+1} \circ \rho$. In particular, $\rho\left(F_{j}\right)=$ 

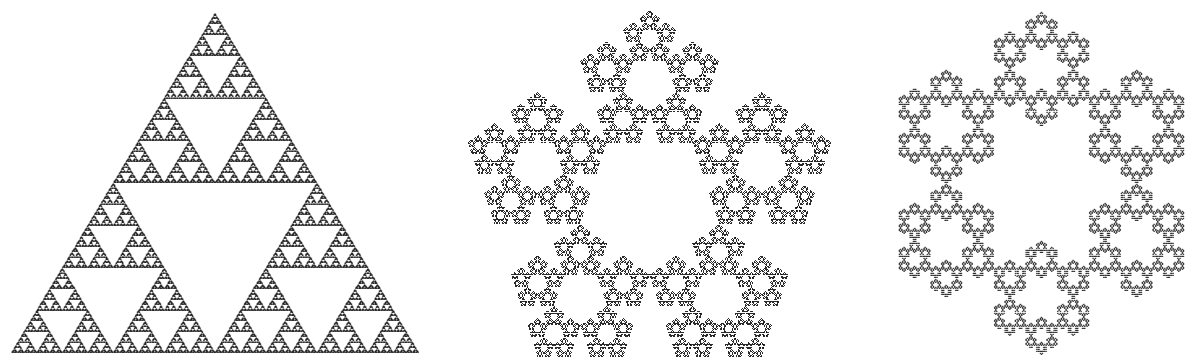

Figure 1. Examples of polygaskets: Sierpiński gasket, pentagasket, and hexagasket, which are respectively a $(1,1,1)$-gasket, a $(1,2,2)$-gasket, and a $(2,2,2)$-gasket.
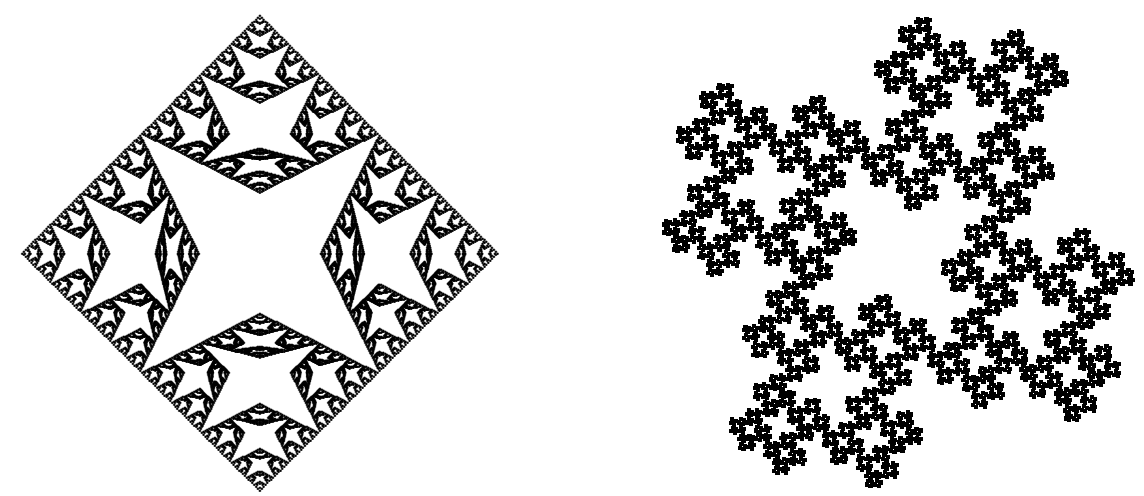

Figure 2. Examples of 4-gaskets: fractal cut square and fractal red cross, which are respectively a $(2,1,1)$-gasket and a $(1,1,2)$ gasket.

$F_{j+1}, \rho\left(v_{j}\right)=v_{j+1}$, and $\left\{\rho^{k}\right\}_{k=1}^{N}$ is a cyclic group of $N$ elements isomorphic to $\mathbb{Z}_{N}=\mathbb{Z} / N \mathbb{Z}$. Without loss of generality, we can assume that $\rho$ is an isometry. An $N$-gasket can be embedded in $\mathbb{R}^{d}$ so that this isometry is a rotation by $2 \pi / N$. Thus, we have:

Proposition 1.2. An $N$-gasket has the dihedral symmetry group $D_{N}$ if and only if $N_{2}=N_{3}$. Otherwise the symmetry group is the cyclic group $\mathbb{Z}_{N}$ of $N$ elements.

We will denote the symmetry group of an $N$-gasket by $\mathscr{G}$.

We are interested in the existence and uniqueness, up to a multiplicative constant, of a self-similar local regular $\mathscr{G}$-invariant resistance (Dirichlet, energy) form $\mathscr{E}$ on any $N$-gasket. By the general results [Kigami 1993; 2001; Metz 1996; 2003; Sabot 1997; Barlow 1998; Hambly et al. 2006; Strichartz 2006], it is enough to prove the existence and uniqueness of a scaling constant $c$ and resistances $R_{k}$, $k=1, \ldots, N-1$, such that the following property holds. 
First, consider a resistor network with vertices $\left\{v_{i}\right\}_{i=1}^{N}$ such that each pair $v_{i}$ and $v_{i+k}$ is connected by a resistance $R_{k}$. We will call this network the 0 -depth network. Note that we use cyclic notation modulo $N$ for indices. Let $R_{k}^{\prime}$ be the effective resistance between $v_{i}$ and $v_{i+k}$, which obviously does not depend on $i$, in this 0-depth network. Second, consider a resistor network with vertices $\left\{v_{i, j}\right\}_{i, j=1}^{N}$ such that each pair $v_{i, j}$ and $v_{i, j+k}$ is connected by a resistance $R_{k}$, and pairs of distinct vertices in different 1 -cells are not directly connected. We will call this network the 1-depth network. Note we have identified the vertices $v_{k, k+N_{3}}$ and $v_{k+1, k+1+N-N_{2}}$. Let $R_{k}^{\prime \prime}$ be the effective resistance between $v_{i}$ and $v_{i+k}$ in this second 1-depth network, which again does not depend on $i$. These two networks are illustrated in Figures 3 and 4. The existence and uniqueness of a self-similar

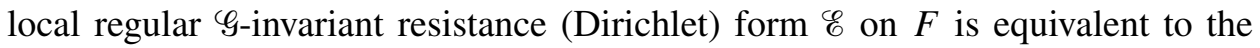
existence and uniqueness of a resistance scaling constant $c$ and resistances $R_{k}$, $k=1, \ldots, N-1$, such that

$$
R_{k}^{\prime \prime}=c R_{k}^{\prime} \quad \text { for all } k=1, \ldots, N .
$$

In other terms, let $V_{0}=\left\{v_{i}\right\}_{i=1}^{N} \subset V_{1}=\left\{v_{i, j}\right\}_{i, j=1}^{N}$. On $V_{0}, V_{1}$, we respectively define finite-dimensional $\mathscr{G}_{\text {-symmetric Dirichlet forms }}$

$$
\begin{aligned}
& \mathscr{E}_{0}(f, f)=\sum_{i \neq j} \frac{1}{R_{i-j}}\left(f\left(v_{i}\right)-f\left(v_{j}\right)\right)^{2}, \\
& \mathscr{E}_{1}(g, g)=\sum_{k=1}^{N} \sum_{i \neq j} \frac{1}{R_{i-j}}\left(g\left(v_{k, i}\right)-g\left(v_{k, j}\right)\right)^{2} .
\end{aligned}
$$

Then our main equation, which is equivalent to (1-1), is

$$
\mathscr{E}_{0}=c \operatorname{Tr}_{V_{0}} \mathscr{E}_{1}
$$

that is, $\mathscr{E}_{0}$ is the same as the trace of $c \mathscr{E}_{1}$ on $V_{0}$. The trace of Dirichlet forms on subsets is defined in the Appendix.
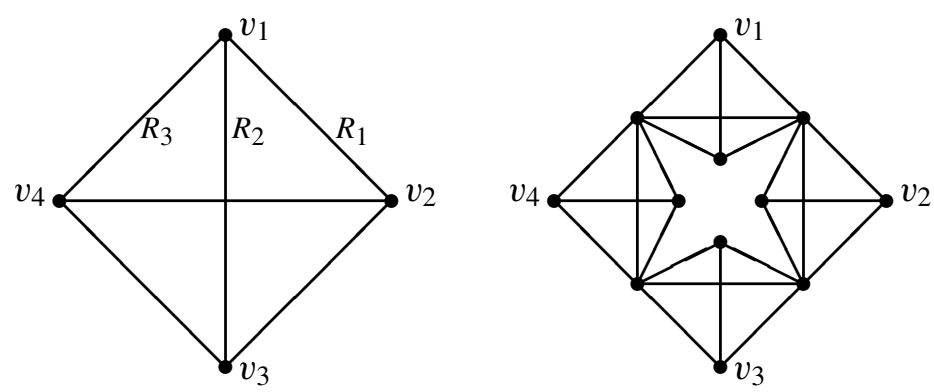

Figure 3. The 0-depth and 1-depth networks in the fractal cut square. 

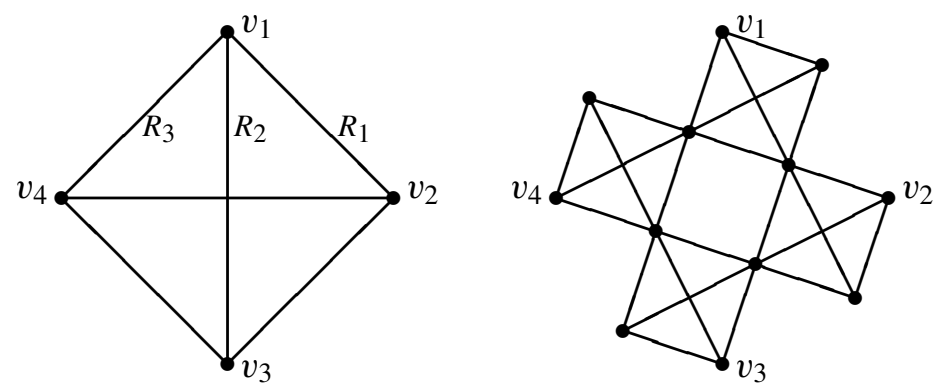

Figure 4. The 0-depth and 1-depth networks in the fractal red cross.

Equations (1-1) and (1-2) allow us to define a local regular self-similar Dirichlet form on $F$ by

$$
\mathscr{E}(f, f)=\lim _{n \rightarrow \infty} c^{n} \sum_{w_{1}, \ldots, w_{n}=1}^{N} \mathscr{E}\left(f \circ \psi_{w_{n}} \ldots \circ \psi_{w_{1}}, f \circ \psi_{w_{n}} \ldots \circ \psi_{w_{1}}\right)
$$

for any $f$ in the domain of the form. The reader can find the general theory of Dirichlet forms in [Bouleau and Hirsch 1991; Fukushima et al. 1994] and the theory of self-similar Dirichlet forms on fractals in [Barlow 1998; Kigami 2001; Strichartz 2006]. According to this theory, on $F$ there exists a densely defined self-adjoint operator $\Delta$, called the Dirichlet self-similar Laplacian on $F$, such that

$$
\mathscr{E}(f, f)=\int_{F} f \Delta f d \mu
$$

for any function in the domain of $\Delta$ that vanishes on the boundary of $F$. Here $\mu$ is the unique self-similar balanced probability measure on $F$ that has

$$
\int_{F} f d \mu=\frac{1}{N} \sum_{i=1}^{N} \int_{F} f \circ \psi_{i} d \mu
$$

for any bounded Borel-measurable function $f$.

To any resistance form there corresponds the so-called effective resistance metric

$$
R_{\mathrm{eff}}(p, q)=\sup \left\{\frac{(u(p)-u(q))^{2}}{\mathscr{E}(u, u)}: u \in \operatorname{Dom}_{\mathscr{E}} \mathscr{E} \mathscr{E}(u, u)>0\right\} .
$$

This metric is studied in detail in [Kigami 1994; 2001]. In particular, it is not self-similar, but asymptotically self-similar with scaling constant $c$. The effective resistance metric is not related to a particular embedding of the fractal into $\mathbb{R}^{n}$, but rather to its intrinsic structure.

Remark 1.3. The resistance scaling constant $c$ is important, in particular, because it allows to compute the Hausdorff dimension of the fractal with respect to 
the effective resistance metric. According to [Kigami and Lapidus 1993; Kigami 1994; 2001], the Hausdorff dimension of the $N$-gaskets with respect to the effective resistance metric is

$$
d_{H}=\frac{\log N}{\log c} .
$$

Note that this dimension has no relation with the embedding of the fractals into $\mathbb{R}^{2}$ in Figures 1 and 2, and it does not correspond to the "usual" dimension of selfsimilar fractals in $\mathbb{R}^{n}$. It rather depends on the "inner" structure of the fractal and on how parts of the fractal are connected.

By definition, the spectral dimension of the Laplacian is equal to

$$
d_{S}=2 \lim _{x \rightarrow \infty} \frac{\log (\rho(x))}{\log (x)},
$$

if the limit exists, where $\rho(x)$ is the eigenvalue counting function of the Laplacian. The spectral dimension is not a dimension in the usual sense of topology or geometric measure theory, but just the exponent that determines the growth rate of the eigenvalue counting function of the Laplacian. However, it is called dimension because, for the usual Laplacian on a compact Riemannian $d$-dimensional manifold or a domain in $\mathbb{R}^{d}$, the spectral dimension is equal to the topological and metric dimension $d$. According to [Kigami and Lapidus 1993; Kigami 2001], for the standard Laplacian on a p.c.f. self-similar set we can compute the spectral dimension by the formula

$$
d_{S}=\frac{2 d_{H}}{d_{H}+1}=\frac{2 \log N}{\log (N c)} .
$$

According to [Strichartz 2003; 2006], this formula shows that the Laplacian is an operator of order $d_{H}+1$.

Note that the spectral dimension $d_{S}$ is not equal to the Hausdorff dimension $d_{H}$ unless both are one. These dimensions for any $N$-gasket can be computed using Theorem 3.2, and their asymptotic behaviors under different assumptions are given in Corollary 5.8.

\section{Transformed $N$-gaskets}

The equivalent Equations (1-1) and (1-2) can be considered a nonlinear $(N-1)$ dimensional eigenvalue problem, which is difficult to solve in general [Metz 1996; 2003; Sabot 1997; Hambly et al. 2006]. To achieve our results we have to change self-similarity structure of $F$ and apply various transformations to the networks involved.

We define

$$
\widetilde{\psi}_{j}=\rho^{j-1} \circ \psi_{1}
$$


and note that by definition $\widetilde{\psi}_{j}(F)=F_{j}$ and $\widetilde{\psi}_{j}\left(v_{k}\right)=v_{j+k-1}$. This means

$$
\widetilde{\psi}_{k}\left(v_{1}\right)=v_{k}, \quad \widetilde{\psi}_{k}\left(v_{N_{3}+1}\right)=v_{k, k+N_{3}}, \quad \widetilde{\psi}_{k}\left(v_{N-N_{2}+1}\right)=v_{k, k+N-N_{2}} .
$$

It is important that the vertices on the left side of these three formulas do not depend on $k$. Thus, we can consider the three vertices $v_{1}, v_{N_{3}+1}, v_{N-N_{2}+1}$ as the boundary of $F$ if we use the self-similar structure of contractions $\widetilde{\psi}_{1}, \ldots, \widetilde{\psi}_{N}$. For simplicity we will let

$$
q_{1}=v_{1}, \quad q_{2}=v_{N_{3}+1}, \quad q_{3}=v_{N-N_{2}+1} .
$$

Based on this construction, it is enough to begin with the network of three vertices $q_{1}, q_{2}, q_{3}$ and three resistances $R_{q_{i}, q_{j}}$ between them. We transform this network into another network of four vertices and three resistances $r_{1}, r_{2}, r_{3}$ by the well-known basic technique from elementary circuit theory known as the $\Delta$-Y transform given by

$$
r_{j}=\frac{R_{q_{j}, q_{j+1}} R_{q_{j}, q_{j-1}}}{R_{q_{1}, q_{2}}+R_{q_{1}, q_{3}}+R_{q_{2}, q_{3}}}
$$

and illustrated in Figure 5.

As the first consequence of these transformations we obtain:

Lemma 2.1. For each $k=1, \ldots, N-1$, we have $R_{k}=R_{N-k}$.

Note that these symmetries of the resistances do not correspond to any symmetries of the $N$-gasket if $N_{2} \neq N_{3}$. Also note that $r_{2} \neq r_{3}$ if $N_{2} \neq N_{3}$.

Proof of Lemma 2.1. One can see that in Figure 8 (see also Figures 6 and 7 as examples) the resistances $r_{2}$ and $r_{3}$ are connected in pairs to form a resistances $r_{2}+r_{3}$. Therefore in the network shown in Figure 8 on the right hand side, the resistance between $v_{i}$ and $v_{i+k}$ is the same as the resistance between $v_{i}$ and $v_{i-k}$. This implies $R_{k}=R_{N-k}$ for $k=1, \ldots, N-1$.
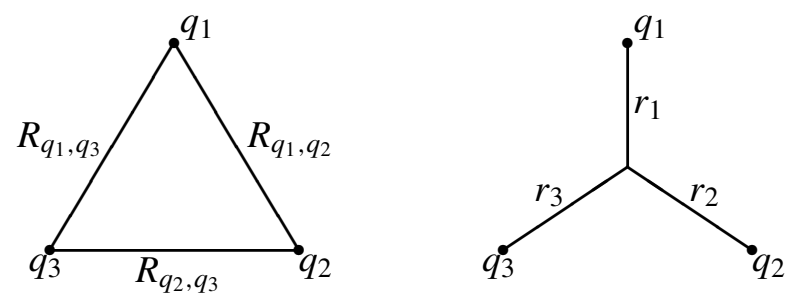

Figure 5. Illustration for the $\Delta-Y$ transform (2-3). 

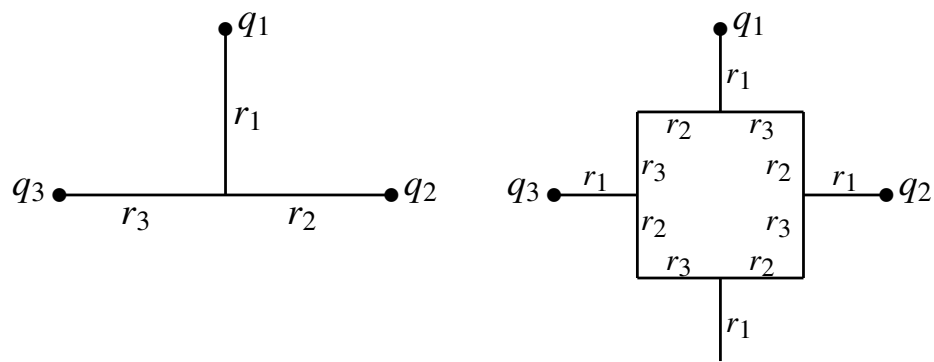

Figure 6. The transformed 0-depth and 1-depth networks in the fractal cut square.

\section{Computations in a transformed $N$-gasket}

Theorem 3.1. Initial resistances $r_{1}, r_{2}, r_{3}$ correspond to a self-similar energy form on the $\left(N_{1}, N_{2}, N_{3}\right)$-gasket if and only if there is a constant $c$ such that

$$
\begin{aligned}
c r_{1} & =r_{1}+\frac{N_{2} N_{3}}{N}\left(r_{2}+r_{3}\right), \\
c r_{2} & =r_{1}+\frac{N_{1} N_{3}}{N}\left(r_{2}+r_{3}\right), \\
c r_{3} & =r_{1}+\frac{N_{1} N_{2}}{N}\left(r_{2}+r_{3}\right) .
\end{aligned}
$$

Proof. The network in Figure 8 is the same as the network in Figure 9 if we are concerned with the effective resistances between the three points $q_{1}, q_{2}, q_{3}$. In the latter network we do the $\Delta-Y$ transform (2-3) with the triangle of resistances $N_{3}\left(r_{2}+r_{3}\right), N_{2}\left(r_{2}+r_{3}\right)$, and $N_{1}\left(r_{2}+r_{3}\right)$. Thus, the result follows from (1-1) and (2-3).
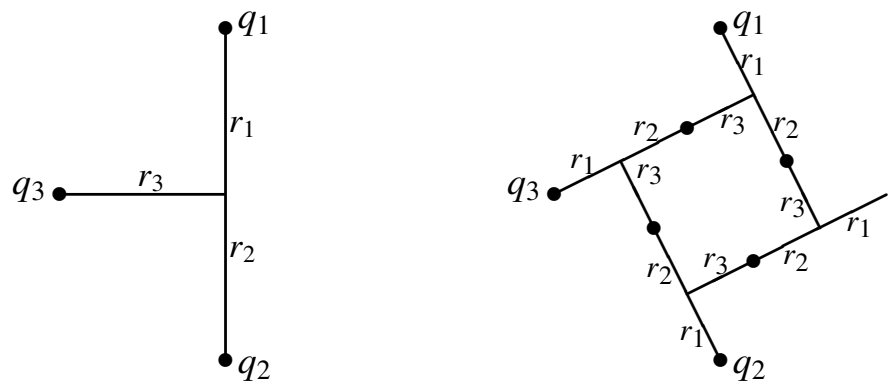

Figure 7. The transformed 0-depth and 1-depth networks in the fractal red cross. 

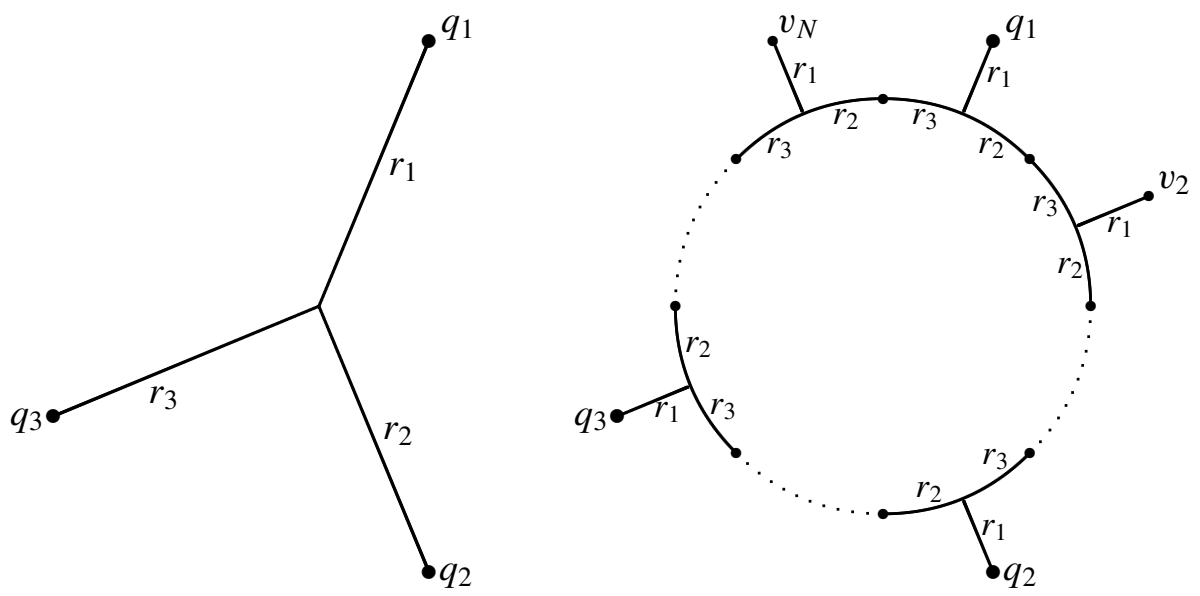

Figure 8. The transformed 0-depth and 1-depth networks for large $N$.

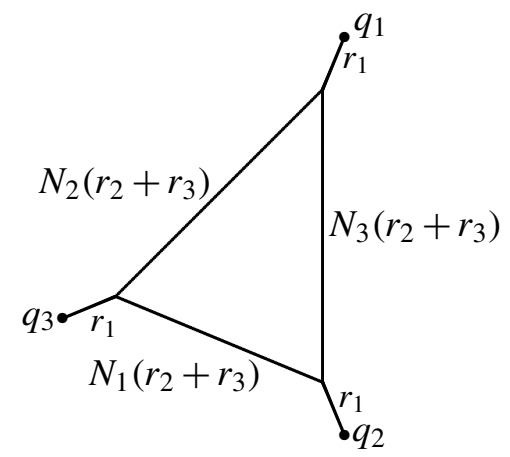

Figure 9. Illustration for the proof of Theorem 3.1.

Theorem 3.2. Let $s=N+N_{1} N_{3}+N_{1} N_{2}$. Then,

$$
c=\frac{s+\sqrt{s^{2}+\left(2 N_{2} N_{3}-N_{1} N_{3}-N_{1} N_{2}\right) 4 N}}{2 N} .
$$

Proof. By (3-4) we have

$$
r_{1}=\frac{1}{(c-1)} \frac{N_{2} N_{3}\left(r_{2}+r_{3}\right)}{N},
$$

which we then plug into (3-5) and (3-6) and obtain

$$
\begin{aligned}
& c r_{2}=\frac{1}{(c-1)} \frac{N_{2} N_{3}\left(r_{2}+r_{3}\right)}{N}+\frac{N_{1} N_{3}\left(r_{2}+r_{3}\right)}{N}, \\
& c r_{3}=\frac{1}{(c-1)} \frac{N_{2} N_{3}\left(r_{2}+r_{3}\right)}{N}+\frac{N_{1} N_{2}\left(r_{2}+r_{3}\right)}{N} .
\end{aligned}
$$


Then, by adding these two lines and dividing by $r_{2}+r_{3}$, we obtain a quadratic equation for $c$ :

$$
N c^{2}-\left(N+N_{1} N_{3}+N_{1} N_{2}\right) c+N_{1} N_{3}+N_{1} N_{2}-2 N_{2} N_{3}=0 .
$$

This implies the result since it is easy to see that $c>1$, which also follows from the general theory [Kigami 2001].

This result is simplified in the next situation.

Corollary 3.3. If $N_{1}=N_{2}=N_{3}=n$, then $c=1+2 n / 3$.

The case $n=1$ corresponds to the Sierpiński gasket, and $n=2$ corresponds to the hexagasket, see Example 3.7 and Figure 1. The larger values of $n$ do not correspond to self-similar fractals in $\mathbb{R}^{2}$, but any $(n, n, n)$-can be constructed as an abstract metric space.

Now that we have solved for $c$, we can find values for $r_{1}, r_{2}, r_{3}$. Note that the resistances are defined up to a scalar multiplier, and so we have to choose a normalization. The simplest answer is obtained with the normalization $r_{1}=1$, and also we obtain the formulas with normalizations $r_{2}+r_{3}=1$ and $r_{1}+r_{2}+r_{3}=1$ as corollaries.

Theorem 3.4. For any normalization, we have for the $c$ is given by (3-7) that

$$
\begin{aligned}
& r_{2}=\frac{r_{1}}{c}\left(1+(c-1) \frac{N_{1}}{N_{2}}\right), \\
& r_{3}=\frac{r_{1}}{c}\left(1+(c-1) \frac{N_{1}}{N_{3}}\right) .
\end{aligned}
$$

Proof. Using Equation (3-4), we get $r_{2}+r_{3}=N(c-1) r_{1} /\left(N_{2} N_{3}\right)$, and so, from (3-5) and (3-6),

$$
c r_{2}=r_{1}+\frac{(c-1)}{N_{1} N_{2}} r_{1}, \quad c r_{3}=r_{1}+\frac{N(c-1)}{N_{1} N_{3}} r_{1} .
$$

Corollary 3.5. When $r_{2}+r_{3}=1$, we have for the $c$ of (3-7) that

$$
\begin{aligned}
& r_{1}=\frac{N_{2} N_{3}}{N(c-1)}, \\
& r_{2}=\frac{N_{2} N_{3}+N_{1} N_{3}(c-1)}{N c(c-1)}, \\
& r_{3}=\frac{N_{2} N_{3}+N_{1} N_{2}(c-1)}{N c(c-1)} .
\end{aligned}
$$

Proof. Using Equation (3-4) and $r_{2}+r_{3}=1$ we obtain $c r_{1}=r_{1}+N_{2} N_{3} / N$, which is (3-10). Then (3-11) and (3-12) follows from (3-5) and (3-6), again using the normalization $r_{2}+r_{3}=1$. 
Corollary 3.6. When $r_{1}+r_{2}+r_{3}=1$ we have

$$
r_{1}=\frac{N_{2} N_{3}}{N(c-1)+N_{2} N_{3}},
$$

where $c$ is given by (3-7), and $r_{2}, r_{3}$ can be computed by (3-8) and (3-9).

Proof. If $r_{2}+r_{3}=1-r_{1}$ then (3-4) immediately implies the result.

Example 3.7. - For the Sierpiński gasket in Figure 1, which is a $(1,1,1)$-gasket, we have

$$
c=\frac{5}{3} \quad \text { and } \quad r_{1}=r_{2}=r_{3} \text {. }
$$

- For the pentagasket in Figure 1, which is a $(1,2,2)$-gasket, we have

$$
c=\frac{9+\sqrt{161}}{10} \text { and } \quad r_{2}=r_{3}=\frac{\sqrt{161}-1}{16} r_{1} .
$$

- For the hexagasket in Figure 1, which is a $(2,2,2)$-gasket, we have

$$
c=\frac{7}{3} \quad \text { and } \quad r_{1}=r_{2}=r_{3}
$$

- For the fractal cut square in Figure 2, which is a $(2,1,1)$-gasket, we have

$$
c=\frac{2+\sqrt{2}}{2} \text { and } \quad r_{2}=r_{3}=\sqrt{2} r_{1} \text {. }
$$

- For the fractal red cross in Figure 2, which is a $(1,1,2)$-gasket, we have

$$
c=\frac{7+\sqrt{65}}{8} \text { and } r_{2}=r_{1}, \quad r_{3}=\frac{2 \sqrt{65}-5}{4} r_{1} .
$$

\section{Resistances of the $N$-gasket using matrix computations}

Having computed the resistance scaling factor $c$ as well the resistances $r_{1}, r_{2}, r_{3}$, we are now interested in the resistances $R_{m}$ between points $v_{k}$ and $v_{k+m}$. Note that the $\Delta$-Y transform allows to compute effective resistances between points $v_{k}$ and $v_{k+m}$, but does not allow to compute $R_{m}$. Instead we use a method where we first compute certain harmonic functions, and then the resistances (see the Appendix).

Notation 4.1. Let $j_{1}, \ldots, j_{N}$ be the junction points between resistances $r_{1}, r_{2}, r_{3}$, as indicated in Figure 10. In particular, each point $j_{k}$ is connected to the boundary point $v_{k}$ by a resistance $r_{1}$. Let $h$ be a harmonic function with boundary values $h\left(v_{1}\right)=1$ and $h\left(v_{k}\right)=0$ for $k=2, \ldots, N$. Then we define $J_{k}=h\left(j_{k}\right)$ for each $k=1, \ldots, N$. 
Lemma 4.2. If $k \neq 1$ then

$$
J_{k} \frac{1}{r_{1}}+\left(\left(J_{k}-J_{k+1}\right)+\left(J_{k}-J_{k+1}\right)\right) \frac{1}{r_{2}+r_{3}}=0
$$

and

$$
\left(J_{1}-1\right) \frac{1}{r_{1}}+\left(J_{1}-J_{2}\right) \frac{1}{r_{2}+r_{3}}+\left(J_{1}-J_{N}\right) \frac{1}{r_{2}+r_{3}}=0 .
$$

Proof. These are the equations of a harmonic function.

We then rewrite the above equations:

Lemma 4.3. Let

$$
z=2+\frac{r_{2}+r_{3}}{r_{1}}
$$

where

$$
\frac{r_{2}+r_{3}}{r_{1}}=\frac{1}{c}\left(1+(c-1) N_{1} \frac{N_{2}+N_{3}}{N_{2} N_{3}}\right)
$$

and $c$ is given by (3-7). Then

$$
J_{N}=J_{2}=-J_{2}+z J_{1}-\frac{r_{2}+r_{3}}{r_{1}},
$$

and, if $k=0, \ldots, N-1$,

$$
\left[\begin{array}{l}
J_{k+1} \\
J_{k+2}
\end{array}\right]=\left[\begin{array}{cc}
0 & 1 \\
-1 & z
\end{array}\right]^{k}\left[\begin{array}{l}
J_{1} \\
J_{2}
\end{array}\right] .
$$

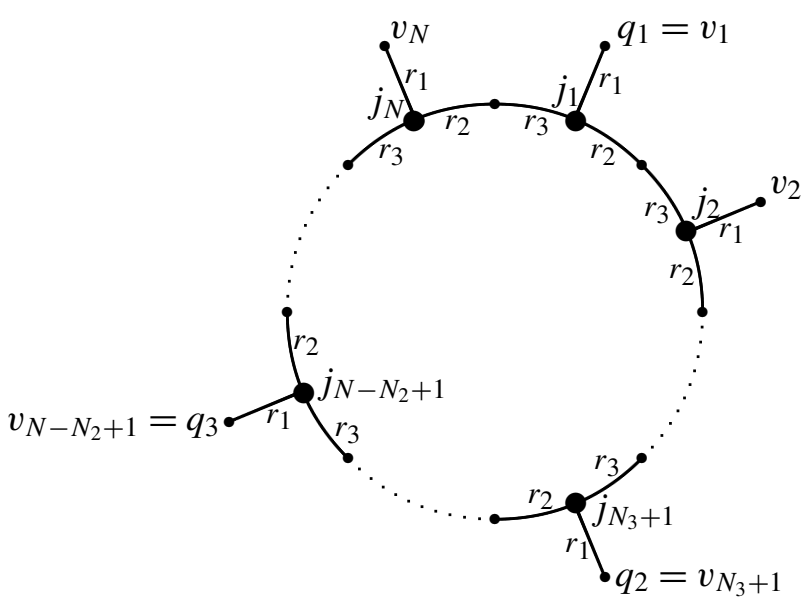

Figure 10. Notation 4.1 for the junction points in the transformed 1-depth network. 
Proof. These are just transformed equations of the previous lemma. Note that $J_{2}=J_{N}$ since the harmonic function is unique and the network in Figure 10 is symmetric around $j_{1}$. More exactly, resistances $r_{2}$ and $r_{3}$ are not symmetrically placed, but in this set-up we are interested only in the joint resistance $r_{2}+r_{3}$ between each pair of points $j_{k}$ and $j_{k+1}$ (see also Lemma 2.1). Note that $J_{N+1}$ is the same as $J_{1}$ by Notation 1.1 .

Next, we will find the powers of the matrix

$$
M=\left[\begin{array}{cc}
0 & 1 \\
-1 & z
\end{array}\right]
$$

\section{Lemma 4.4.}

$$
M^{k}=\frac{1}{\lambda_{+}-\lambda_{-}}\left[\begin{array}{cc}
\lambda_{-}^{k-1}-\lambda_{+}^{k-1} & \lambda_{+}^{k}-\lambda_{-}^{k} \\
\lambda_{-}^{k}-\lambda_{+}^{k} & \lambda_{+}^{k+1}-\lambda_{-}^{k+1}
\end{array}\right],
$$

in which $\lambda_{+}, \lambda_{-}$are the eigenvalues of $M$ :

$$
\lambda_{ \pm}=\frac{z \pm \sqrt{z^{2}-4}}{2} .
$$

Note that both $\lambda_{+}$and $\lambda_{-}$are real, since $z>2$.

Proof. First, we find the eigenvectors of the matrix $M$, that is we solve for $M V_{+}=$ $\lambda_{+} V_{+}$and $M V_{-}=\lambda_{-} V_{-}$. Then we can find $M^{k}=C D^{k} C^{-1}$ where

$$
D=\left[\begin{array}{cc}
\lambda_{+}^{k} & 0 \\
0 & \lambda_{-}^{k}
\end{array}\right]
$$

and $C=\left[V_{+}, V_{-}\right]$is the matrix made from the eigenvectors of the matrix $M$. The matrix $C$ is not unique, and the easiest way to write it is

$$
C=\left[\begin{array}{cc}
1 & 1 \\
\lambda_{+} & \lambda_{-}
\end{array}\right]
$$

and

$$
C^{-1}=\frac{1}{\lambda_{+}-\lambda_{-}}\left[\begin{array}{cc}
-\lambda_{-} & 1 \\
\lambda_{+} & -1
\end{array}\right]
$$

Thus,

$$
M^{k}=\frac{1}{\lambda_{+}-\lambda_{-}}\left[\begin{array}{cc}
1 & 1 \\
\lambda_{+} & \lambda_{-}
\end{array}\right]\left[\begin{array}{cc}
\lambda_{+}^{k} & 0 \\
0 & \lambda_{-}^{k}
\end{array}\right]\left[\begin{array}{cc}
-\lambda_{-} & 1 \\
\lambda_{+} & -1
\end{array}\right]
$$


Simplifying this equation, we get

$$
\begin{aligned}
M^{k} & =\frac{1}{\lambda_{+}-\lambda_{-}}\left[\begin{array}{cc}
\lambda_{+}^{k} & \lambda_{-}^{k} \\
\lambda_{+}^{k+1} & \lambda_{-}^{k+1}
\end{array}\right]\left[\begin{array}{cc}
-\lambda_{-} & 1 \\
\lambda_{+} & -1
\end{array}\right] \\
& =\frac{1}{\lambda_{+}-\lambda_{-}}\left[\begin{array}{cc}
-\lambda_{+}^{k} \lambda_{-}+\lambda_{+} \lambda_{-}^{k} & \lambda_{+}^{k}-\lambda_{-}^{k} \\
-\lambda_{+}^{k+1} \lambda_{-}+\lambda_{+} \lambda_{-}^{k+1} & \lambda_{+}^{k+1}-\lambda_{-}^{k+1}
\end{array}\right] .
\end{aligned}
$$

Noting that $\lambda_{+} \lambda_{-}=1$, the lemma is proved.

From the previous two lemmas we obtain a corollary. Remember that in our notation $J_{N+1}=J_{1}$.

Corollary 4.5. For $k=2, \ldots, N+1$,

$$
J_{k}=\frac{1}{\lambda_{+}-\lambda_{-}}\left(\left(\lambda_{-}^{k-2}-\lambda_{+}^{k-2}\right) J_{1}+\left(\lambda_{+}^{k-1}-\lambda_{-}^{k-1}\right) J_{2}\right) .
$$

Theorem 4.6.

$$
J_{1}=\left(\frac{r_{2}+r_{3}}{r_{1}}\right)\left(\frac{\lambda_{+}^{N}-\lambda_{-}^{N}}{2\left(\lambda_{-}^{N-1}-\lambda_{+}^{N-1}\right)+\left(\lambda_{+}^{N}-\lambda_{-}^{N}\right) z-2\left(\lambda_{+}-\lambda_{-}\right)}\right)
$$

and

$$
J_{2}=\frac{1}{2}\left(z J_{1}-\frac{r_{2}+r_{3}}{r_{1}}\right),
$$

where $z,\left(r_{2}+r_{3}\right) / r_{1}$, and $\lambda_{ \pm}$are given by formulas (4-13), (4-14), (4-19).

Proof. Equation (4-25) follows from (4-15). Then, given that $M^{N-1}=\left[\begin{array}{ll}A & B \\ C & D\end{array}\right]$, we have

$$
\left[\begin{array}{c}
J_{N} \\
J_{N+1}
\end{array}\right]=\left[\begin{array}{ll}
A & B \\
C & D
\end{array}\right]\left[\begin{array}{l}
J_{1} \\
J_{2}
\end{array}\right] \text {. }
$$

However, we know that $J_{N+1}=J_{1}$. So after matrix multiplication we get that $J_{1}=C J_{1}+D J_{2}$. Substitution of $J_{2}$ gives

$$
J_{1}=C J_{1}+\frac{1}{2} D\left(z J_{1}-\frac{r_{2}+r_{3}}{r_{1}}\right), \quad \text { that is, } \quad J_{1}=\frac{r_{2}+r_{3}}{r_{1}} \frac{D}{2 C+z D-2} .
$$

Then (4-24) follows from (4-18).

Remark 4.7. Note that the values $J_{k}$ are important since they allow to compute so-called harmonic matrices, that is, transformations $h \mapsto h \circ \psi_{i}$ acting on the space of harmonic functions [Kigami 2001; Strichartz 2006; Teplyaev $\geq 2007$ ].

Recall that, by definition, $R_{m}$ are the resistances between the boundary points $v_{1}$ and $v_{m+1}$ in the $N$-gasket. The set of these resistances is defined uniquely up to a constant multiplier. It seems the most convenient to normalize these resistances so that $R_{1}=1$. 
Lemma 4.8. Up to a multiplicative constant, which depends on the normalization, we have $R_{m}=r_{1} / J_{m+1}$, where the $J_{m+1}$ is defined by (4-23).

Proof. According to Notation 4.1, the harmonic function $h$ of this section is the same as the harmonic function $h_{y}$ considered in the Appendix if $y=v_{1}$. Moreover, by definition $R_{m}$ is the resistance between $v_{1}$ and $v_{m+1}$ in the network, which is the trace of the network in Figure 10 on the boundary $\left\{v_{1}, \ldots, v_{N}\right\}$. Therefore $1 / R_{m}=C_{v_{1}, v_{m+1}}^{\prime}$ in the notation of Theorem A.10. Therefore, by Theorem A.10 with $x=v_{m+1}$, we have

$$
\frac{1}{R_{m}}=C_{v_{m+1}, j_{m+1}} h\left(j_{m+1}\right)=\frac{1}{r_{1}} J_{m+1} .
$$

Note that in this case the sum in the second formula in Theorem A.10 has only one nonzero term with $p=j_{m+1}$.

Theorem 4.9. If the normalization of resistances is such that $R_{1}=1$, then

$$
R_{m}=\frac{\lambda_{+}-\lambda_{-}}{\lambda_{+}^{m}-\lambda_{-}^{m}+\frac{J_{1}}{J_{2}}\left(\lambda_{-}^{m-1}-\lambda_{+}^{m-1}\right)},
$$

where $\lambda_{ \pm}, J_{1}$, and $J_{2}$ are given by formulas (4-19), (4-24), and (4-25).

Proof. According to Lemma 4.8, we have

$$
R_{m}=\mathrm{const} \frac{r_{1}}{J_{m+1}}
$$

where $J_{m+1}$ is given by (4-23) and const is a normalization constant. We choose this constant by the condition $R_{1}=1$.

\section{Asymptotic behavior}

Here we study the asymptotics of the resistances $R_{m}$ and the resistance scaling factor $c$ as $N_{1}, N_{2}, N_{3}$ tend to infinity. More precisely, we assume that $N_{1}(n), N_{2}(n)$, $N_{3}(n)$ depend on a parameter $n$ in a given way and consider various asymptotics as $n \rightarrow \infty$. Our main result in this section is:

Theorem 5.1. Assume that

$$
N_{1}(n)=\alpha n+o(n)_{n \rightarrow \infty}, \quad N_{2}(n)=\beta n+o(n)_{n \rightarrow \infty}, \quad N_{3}(n)=\gamma n+o(n)_{n \rightarrow \infty},
$$

where $\alpha, \beta, \gamma$ are positive constants. Then we have the limits

$$
\lim _{n \rightarrow \infty} \frac{c(n)}{n}=\frac{\alpha(\beta+\gamma)}{\alpha+\beta+\gamma}
$$


and

$$
\lim _{n \rightarrow \infty} R_{m}(n)=R_{m, \infty}=\lambda_{+, \infty}^{|m|-1},
$$

where

$$
\lambda_{+, \infty}=\frac{z_{\infty}+\sqrt{z_{\infty}^{2}-4}}{2} \text { and } z_{\infty}=2+\alpha \frac{\beta+\gamma}{\beta \gamma} .
$$

(Note that the set of resistances $\left\{R_{m}(n)\right\}$ is defined up to a scalar multiplier, and for convenience we use the normalization with $R_{1}(n)=1$.)

Note also that in our notation $R_{-1}=R_{N-1}, R_{-2}=R_{N-2}$, etc.

Proof. Formula (5-28) follows from (3-7) since

$$
N(n)=(\alpha+\beta+\gamma) n+o(n)_{n \rightarrow \infty}, \quad \text { and } \quad s(n)=\alpha \gamma n^{2}+\alpha \beta n^{2}+o\left(n^{2}\right)_{n \rightarrow \infty} .
$$

Then from, (3-10) and (4-13), we obtain, with the normalization $r_{2}+r_{3}=1$, the limits

$$
\lim _{n \rightarrow \infty} r_{1}(n)=\frac{\beta \gamma}{\alpha(\beta+\gamma)}=r_{1, \infty} \quad \text { and } \quad \lim _{n \rightarrow \infty} z(n)=\frac{\alpha \beta+2 \beta \gamma+\alpha \gamma}{\beta \gamma}=z_{\infty} .
$$

Hence we also have the limits

$$
\lim _{n \rightarrow \infty} \lambda_{ \pm}(n)=\frac{z_{\infty} \pm+\sqrt{z_{\infty}^{2}-4}}{2}=\lambda_{ \pm+, \infty}
$$

Moreover,

$$
\begin{aligned}
& \lim _{n \rightarrow \infty} J_{1}(n)=\frac{\alpha(\beta+\gamma)}{\beta \gamma \sqrt{z_{\infty}^{2}-4}}=J_{1, \infty}, \\
& \lim _{n \rightarrow \infty} J_{2}(n)=\frac{\alpha(\beta+\gamma)}{2 \beta \gamma}\left(\frac{z}{\sqrt{z_{\infty}^{2}-4}}-1\right)=\lambda_{-, \infty} J_{1, \infty}=J_{2, \infty},
\end{aligned}
$$

which imply $\lim _{n \rightarrow \infty} J_{k}(n)=\lambda_{-, \infty}^{|k-1|} J_{1, \infty}$ for $k \geqslant 0$; recall that in our notation $J_{0}=J_{N}, J_{-1}=J_{N-1}$ etc. Then (5-29) follows from (4-27).

Remark 5.2. We use a specific normalization for the resistances. With the normalization $r_{2}+r_{3}=1$, we have

$$
\lim _{n \rightarrow \infty} R_{m}(n)=\frac{\beta^{2} \gamma^{2} \sqrt{z_{\infty}^{2}-4}}{\alpha^{2}(\beta+\gamma)^{2}} \lambda_{+, \infty}^{|m|}=\lambda_{+, \infty}^{|m|-1} R_{1, \infty}
$$

for $m>0$. The formula

$$
\lim _{n \rightarrow \infty} R_{m}(n)=\lambda_{+, \infty}^{|m|-1} R_{1, \infty}
$$

is also true for normalizations $r_{1}=1$ and $r_{1}+r_{2}+r_{3}=1$. 
Corollary 5.3. Assume that $N_{1}=N_{2}=N_{3}=n \rightarrow \infty$. Then, with normalization $R_{1}=1$, we have the limits

$$
\lim _{n \rightarrow \infty} \frac{c(n)}{n}=\frac{2}{3} \quad \text { and } \quad \lim _{n \rightarrow \infty} R_{m}(n)=(2+\sqrt{3})^{|m|-1} .
$$

Corollary 5.4. Assume that the $N$-gasket $F$ is a polygasket. Then, with normalization $R_{1}=1$, we have the limits

$$
\lim _{n \rightarrow \infty} \frac{c(n)}{n}=1 \quad \text { and } \quad \lim _{n \rightarrow \infty} R_{m}(n)=(3+2 \sqrt{3})^{|m|-1} .
$$

Proof. It is easy to obtain from elementary geometry [Strichartz 1999b; 2000; 2006; Tyson and Wu 2005] that an $N$-gasket is a polygasket if and only if $N$ is not divisible by 4 and $N_{2}=N_{3}=[N / 4]+1$, where [.] means the integer part of a number.

Theorem 5.5. Assume that

$$
N_{1}(n)=\alpha, \quad N_{2}(n)=\beta n+o(n)_{n \rightarrow \infty}, \quad N_{3}(n)=\gamma n+o(n)_{n \rightarrow \infty},
$$

where $\alpha, \beta, \gamma$ are positive constants. Then, for any nonzero $n, k$, we have the limits

$$
\lim _{n \rightarrow \infty} \frac{c(n)}{\sqrt{n}}=\sqrt{2 \frac{\beta \gamma}{\beta+\gamma}}, \quad \lim _{n \rightarrow \infty} \frac{R_{m}(n)}{R_{k}(n)}=1 .
$$

Proof. The first limit follows from (3-7) since

$$
N(n)=(\beta+\gamma) n+o(n)_{n \rightarrow \infty} \quad \text { and } \quad s(n)=(\alpha+1)(\beta+\gamma) n+o(n)_{n \rightarrow \infty} .
$$

Then from (4-14) we obtain

$$
\lim _{n \rightarrow \infty} \frac{r_{2}(n)+r_{3}(n)}{r_{1}(n)}=0, \quad \text { which implies } \quad \lim _{n \rightarrow \infty} z(n)=2 .
$$

Hence we also have $\lim _{n \rightarrow \infty} \lambda_{ \pm}(n)=1$. This implies the second limit.

Theorem 5.6. Assume that

$$
N_{1}(n)=\alpha n+o(n)_{n \rightarrow \infty}, \quad N_{2}(n)=\beta n+o(n)_{n \rightarrow \infty}, \quad N_{3}(n)=\gamma,
$$

where $\alpha, \beta, \gamma$ are positive constants. Then, if $|m|>|k|>0$, we have the limits

$$
\lim _{n \rightarrow \infty} \frac{c(n)}{n}=\frac{\alpha \beta}{\alpha+\beta}, \quad \text { and } \quad \lim _{n \rightarrow \infty} \frac{R_{m}(n)}{R_{k}(n)}=0 .
$$

Proof. The first limit follows from (3-7) since

$$
N(n)=(\alpha+\beta) n+o(n)_{n \rightarrow \infty} \quad \text { and } \quad s(n)=\alpha \beta n^{2}+o\left(n^{2}\right)_{n \rightarrow \infty} .
$$


Then we have the second limit after applying (4-14) to obtain

$$
\frac{r_{2}(n)+r_{3}(n)}{r_{1}(n)}=\frac{\alpha}{\gamma} n+o(n)_{n \rightarrow \infty} .
$$

The case

$$
N_{1}(n)=\alpha n+o(n)_{n \rightarrow \infty}, \quad N_{2}(n)=\beta, \quad N_{3}(n)=\gamma n+o(n)_{n \rightarrow \infty}
$$

can be treated analogously.

Theorem 5.7. Assume that

$$
N_{1}(n)=\alpha n+o(n)_{n \rightarrow \infty}, \quad N_{2}(n)=\beta, \quad N_{3}(n)=\gamma,
$$

where $\alpha, \beta, \gamma$ are positive constants. Then, if $|m|>|k|>0$, we have the limits

$$
\lim _{n \rightarrow \infty} c(n)=\beta+\gamma \quad \text { and } \quad \lim _{n \rightarrow \infty} \frac{R_{m}(n)}{R_{k}(n)}=0 .
$$

Proof. The first formula follows from (3-7) since

$$
N(n)=\alpha n+o(n)_{n \rightarrow \infty} \quad \text { and } \quad s(n)=(1+\gamma+\beta) \alpha n+o(n)_{n \rightarrow \infty} .
$$

Then we have the second limit after applying (4-14) to get $\lim _{n \rightarrow \infty} z(n)=\infty$.

Corollary 5.8. For any value of the parameter $n$ we denote the spectral dimension of the Laplacian on the $N$-gasket by $d_{S}(n)$ and the Hausdorff dimension of the $N$ gasket with respect to the effective resistance metric by $d_{H}(n)$, as in Remark 1.3. Then

(i) With the assumptions of Theorem 5.1 or 5.6, we have

$$
\lim _{n \rightarrow \infty} d_{H}(n)=d_{S}(n)=1 ;
$$

(ii) With the assumptions of Theorem 5.5, we have

$$
\lim _{n \rightarrow \infty} d_{H}(n)=2 \quad \text { and } \quad \lim _{n \rightarrow \infty} d_{S}(n)=\frac{4}{3} ;
$$

(iii) With the assumptions of Theorem 5.7 we have

$$
\lim _{n \rightarrow \infty} d_{H}(n)=\infty \quad \text { and } \quad \lim _{n \rightarrow \infty} d_{S}(n)=2 .
$$

Note that formula of (i) applies, in particular, to polygaskets with the number of sides in the corresponding polygon approaching infinity. 


\section{Appendix: Resistor networks, harmonic functions, traces of Dirichlet forms and random walks}

We recall some basic facts of resistor networks (electrical circuits) and their relation to the finite dimensional Dirichlet forms and to random walks. The classical reference on the relation between random walks and discrete potential theory is [Dynkin and Yushkevich 1969]. A newer reference more relevant to the electrical circuits formalism is [Doyle and Snell 1984]. As an expository remark we note that there exists a different use of probabilistic techniques in analysis on fractals, based on Martin and Poisson boundaries; see [Denker and Sato 2002; Koch and Denker 2005] and references therein.

For a single resistor, Ohm's Law is that the voltage $V$, the current $I$ and the resistance $R$ are related by $V=I R$. In addition, the resistor dissipates energy at the rate of

$$
E=V I
$$

In terms of the conductance $C=1 / R$, we have

$$
E=C V^{2} \text {. }
$$

In a resistor network, we have

- the Voltage Law: the total change of voltage over a closed loop is zero;

- the Current Law: at an inner junction point, the same amount of current flows in as out.

These laws imply two basic rules for resistances. If there are two consecutively connected resistances $R_{1}$ and $R_{2}$, also called series resistors, then the total resistance is $R_{1}+R_{2}$. If there are two parallel resistances $R_{1}$ and $R_{2}$, then the total resistance is $R_{1} R_{2} /\left(R_{1}+R_{2}\right)$. Thus if there are two parallel conductances $C_{1}$ and $C_{2}$, then the total conductances is $C_{1}+C_{2}$. Another rule is the $\Delta-Y$ transform (2-3).

In a network we denote $V(x, y)$ the voltage change from $x$ to $y$. Then the Voltage Law implies that there is a function $U(x)$, called the electric potential, such that $V(x, y)=U(x)-U(y)$ for any two points $x, y$ in the network. The potential is unique up to an additive constant. An easy construction of a potential is as follows. Assign some potential $U_{0}$ to some $x_{0}$. Then for another $x$ consider a path $x_{0}, x_{1}, \ldots, x_{n}$ such that $x_{j}$ and $x_{j+1}$ are connected. Then define

$$
U(x)=U_{0}+V\left(x_{0}, x_{1}\right)+V\left(x_{1}, x_{2}\right)+\cdots+V\left(x_{n-1}, x\right) .
$$

The Voltage Law implies that $U(x)$ is the same for different paths from $x_{0}$ to $x$.

The conductance between any two junction points $x$ and $y$ in the network is denoted by $C_{x, y} \geqslant 0$. The conductance $C_{x, y}$ is zero by definition if the points $x$ 
and $y$ are not directly connected. For a given function $f$ defined on junction points of the network, we define its energy by

$$
\mathscr{E}(f, f)=\sum_{x, y} C_{x, y}(f(x)-f(y))^{2} .
$$

Note that, according to (A-1), if $f=U$ is a potential, then the energy $\mathscr{E}$ is the same as the total electrical energy, which is the sum of energies (A-1) over all resistors in the network.

From now on we will denote the set of junction points in the network by $V$, which should not be confused with the voltage mentioned in the beginning of this section. Also, from now on the junction points will be called vertices.

Definition A.9. A function $h$ is called harmonic at $p$ if

$$
\sum_{q \in V} C_{p, q}(h(p)-h(q))=0 .
$$

We call h a harmonic function if it is harmonic at every inner vertex.

It is an easy consequence of Ohm's Law and the Current Law that any electric potential function $U(x)$ is a harmonic function.

We always assume that a network is connected in that for any vertices $x$ and $y$ there is a path $x=x_{0}, x_{1}, \ldots, x_{n}=y$ such that each pair $x_{j}$ and $x_{j+1}$ is connected by a positive conductance.

It is easy to see from the connectedness of the network, the definition of a harmonic function, and the positivity of conductances that the Strong Maximum Principle holds for any harmonic function: a harmonic function can not have a local maximum or minimum at an inner vertex. From this principle one can obtain another important fact about harmonic functions: for any set of boundary values there exists a unique harmonic function with these values. In other terms it means the following. Let $V_{\text {inner }}$ denote the set of inner vertices and $\partial V$ denote its complement in $V$. Naturally $\partial V$ is called the boundary. Then the unique harmonic extension property is that for any function $g$ on the boundary $\partial V$ there is a unique harmonic function $h$ such that $g=\left.h\right|_{\partial V}$. To prove the unique harmonic extension property notice that the equations defining a harmonic function are linear, the number of equations is the same as the number of variables, and the only solution of the homogeneous system is zero by the Strong Maximum Principle.

To get the formula for the harmonic extension, define $h_{y}(x)$ for each $y \in \partial V$ as the unique harmonic function such that $h_{y}(y)=1$ and $h_{y}(x)=0$ for every other $x \in \partial V$. Then the harmonic extension formula is

$$
h(x)=\sum_{y \in \partial V} h(y) h_{y}(x) \quad \text { for any } \quad x \in V .
$$


The last principle is the Minimal Energy Principle: the harmonic function has the minimal energy among the functions with given boundary values. This principle is proved directly by minimizing the energy. The Minimal Energy Principle implies that the Effective conductance between $p$ and $q$ is

$$
C_{\text {effect }}(p, q)=\frac{E(h, h)}{(h(p)-h(q))^{2}},
$$

where $h$ is any nonconstant harmonic function in the network with the boundary values given at $\partial V=\{p, q\}$. The effective resistance is the reciprocal of the effective conductance. Note that here and in other situations the notion of the boundary $\partial V$ of a finite set $V$ is flexible, and can depend on a particular function and on other considerations.

The trace of the resistance form $\mathscr{E}$ on the boundary $\partial V$ is defined as the unique resistance form $\operatorname{Tr}_{\partial V} \mathscr{E}$ such that for any function $g$ on $\partial V$ we have

$$
\operatorname{Tr}_{\partial V} \mathscr{E}(g, g)=\mathscr{E}(h, h),
$$

where $h$ is the unique harmonic extension of $g$. The next theorem is the main result of this section.

\section{Theorem A.10.}

$$
\operatorname{Tr}_{\partial V} \mathscr{E}(g, g)=\sum_{x, y \in \partial V} C_{x, y}^{\prime}(g(x)-g(y))^{2}, \quad \text { where } C_{x, y}^{\prime}=\sum_{p \in V} h_{y}(p) C_{x, p} .
$$

Note that the strong maximum principle implies that $h_{y}(p) \geqslant 0$ and so $C_{x, y}^{\prime} \geqslant 0$.

Proof. For any function $h$ we have

$$
\begin{aligned}
\mathscr{E}(h, h) & =\sum_{x, p \in V} C_{x, p}(h(x)-h(p))^{2} \\
& =\sum_{x, p \in V} C_{x, p}((h(x)(h(x)-h(p))+h(p)(h(p)-h(x))) \\
& =2 \sum_{x, p \in V} h(x) C_{x, p}(h(x)-h(p)) .
\end{aligned}
$$

Let $h$ be the unique harmonic extension to $V$ of a function $g$ defined on $\partial V$. Then $\sum_{p \in V} C_{x, p}(h(x)-h(p))=0$ if $x$ is not a boundary point. Therefore

$$
\mathscr{E}(h, h)=2 \sum_{x \in \partial V, p \in V} h(x) C_{x, p}(h(x)-h(p)) .
$$

For any $p \in V$ we have $\sum_{y \in \partial V} h_{y}(p)=1$, because a function that is identically one on $\partial V$ has a unique harmonic extension that is identically one on $V$. Using 
this and $h(x)=\sum_{y \in \partial V} h(y) h_{y}(x)$, we obtain

$$
\begin{aligned}
\mathscr{E}(h, h) & =2 \sum_{\substack{x \in \partial V \\
p \in V}} h(x) C_{x, p} \sum_{y \in \partial V} h_{y}(p)(h(x)-h(y)) \\
& =2 \sum_{x, y \in \partial V} h(x)(h(x)-h(y)) \sum_{p \in V} C_{x, p} h_{y}(p) \\
& =\sum_{x, y \in \partial V}(h(x)(h(x)-h(y))+h(y)(h(y)-h(x))) \sum_{p \in V} C_{x, p} h_{y}(p) \\
& =\sum_{x, y \in \partial V}(g(x)-g(y))^{2} \sum_{p \in V} C_{x, p} h_{y}(p) .
\end{aligned}
$$

It is well known that Dirichlet forms, resistor networks and symmetric random walks lead to essentially the same harmonic analysis on a finite set $V$ (see for instance [Doyle and Snell 1984]). It is clear that Dirichlet forms and resistor networks are in one-to-one correspondence. A random walk $X_{n}, n=0,1,2, \ldots$, is defined through its transition probabilities $p(x, y), x, y \in V$, which means that $X_{n+1}=y$ with probability $p(x, y)$ if $X_{n}=x$. We next assume that, for $x \neq y$,

$$
p(x, y)=\frac{C_{x, y}}{\sum_{z \in V} C_{x, z}} .
$$

In this situation we say that the resistor network and the random walk are equivalent. We call any random walk so defined symmetric. The symmetric random walks are in one-to-one correspondence with Dirichlet forms up to a constant multiple.

We assume that $\mathscr{E}(f, f)=0$ if and only if $f$ is constant. This means that the random walk $X_{n}$ can reach any point with positive probability in a finite number of steps. In terms of the resistor network, this means that the network does not have more than one connected component.

Let $P_{x}\{\cdot\}$ denote the probability distribution of the random walk $X_{n}$ that starts at $X_{0}=x$, that is $P_{x}\left\{X_{0}=x\right\}=1$, and let $E_{x}$ denote the expectation with respect to $P_{x}\{\cdot\}$. Then a function $h$ is harmonic at $x \in V$ if and only if $h(x)=E_{x} h\left(X_{1}\right)=$ $\sum_{y \in V} p(x, y) h(y)$. Moreover, $h(x)$ can be expressed through its boundary values: Let $\tau$ be the first time $X_{n}$ reaches $\partial V$, that is $\tau=\min \left\{n: X_{n} \in \partial V\right\}$. Then $h(x)=E_{x} h\left(X_{\tau}\right)$ for any $x \in V$. This expression depends only on the values of $h$ on $\partial V$, since $X_{\tau} \in \partial V$ with probability one. In particular, $h_{y}(x)=P_{x}\left\{X_{\tau}=y\right\}$.

Let $p^{\prime}(x, y)=P_{x}\left\{X_{\tau_{x}^{\prime}}=y\right\}$, where $\tau_{x}^{\prime}$ is the first time $X_{n}$ reaches $\partial V \backslash\{x\}$, that is $\tau_{x}^{\prime}=\min \left\{n: X_{n} \in \partial V \backslash\{x\}\right\}$. In other words, the random walk $X_{n}$ that starts at $x \in \partial V$ is killed at $\partial V \backslash\{x\}$. Then $p^{\prime}(x, y)$ is the probability that this walk is killed at $y$. Then it is easy to see from the Markov property that the $p^{\prime}(x, y)$ are the transition probabilities of the random walk on $\partial V$ that corresponds to the Dirichlet form $\operatorname{Tr}_{\partial V} \mathscr{E}$. This is the relation used in [Lindstrøm 1990; Barlow 1998] to define 
self-similar diffusion on the fractals. One can obtain other relations between random walks and resistance forms. For instance, $c=1 /\left(1-p_{x}^{\prime \prime}\right)$, where $p_{x}^{\prime \prime}$ is the probability that the random walk $X_{n}$ that starts at $x \in \partial V$ returns to $x$ before it is killed when it hits $\partial V \backslash\{x\}$.

\section{Acknowledgments}

The authors thank Andrew Polonsky for useful remarks. The last author is grateful to Volker Metz for helpful suggestions and to Michael Levy, Richard Kenyon, and Robert Strichartz for involvement in the early part of this project at Cornell University.

\section{References}

[Adams et al. 2003] B. Adams, S. A. Smith, R. S. Strichartz, and A. Teplyaev, "The spectrum of the Laplacian on the pentagasket", pp. 1-24 in Fractals in Graz 2001, edited by P. Grabner and W. Woess, Trends Math., Birkhäuser, Basel, 2003. MR 2006g:28017 Zbl 1037.31010

[Alexander 1984] S. Alexander, "Some properties of the spectrum of the Sierpiński gasket in a magnetic field", Phys. Rev. B (3) 29:10 (1984), 5504-5508. MR 85m:82076

[Bajorin et al. $\geq 2007$ ] N. Bajorin, T. Chen, A. Dagan, C. Emmons, M. Hussein, M. Khalil, P. Mody, B. Steinhurst, and A. Teplyaev, "Vibration modes of $3 n$-gaskets and other fractals", J. Phys. A. To appear.

[Barlow 1998] M. T. Barlow, "Diffusions on fractals", pp. 1-121 in Lectures on probability theory and statistics (Saint-Flour, 1995), edited by P. Bernard, Lecture Notes in Math. 1690, Springer, Berlin, 1998. MR 2000a:60148 Zbl 0916.60069

[Bouleau and Hirsch 1991] N. Bouleau and F. Hirsch, Dirichlet forms and analysis on Wiener space, de Gruyter Studies in Mathematics 14, Walter de Gruyter \& Co., Berlin, 1991. MR 93e:60107 Zbl 0748.60046

[Denker and Sato 2002] M. Denker and H. Sato, "Reflections on harmonic analysis of the Sierpiński gasket”, Math. Nachr. 241 (2002), 32-55. MR 2003e:28016 Zbl 1020.60066

[Doyle and Snell 1984] P. G. Doyle and J. L. Snell, Random walks and electric networks, Carus Mathematical Monographs 22, Mathematical Association of America, Washington, DC, 1984. MR 89a:94023 Zbl 0583.60065

[Dynkin and Yushkevich 1969] E. B. Dynkin and A. A. Yushkevich, Markov processes: Theorems and problems, Translated from the Russian by James S. Wood, Plenum Press, New York, 1969. MR 39 \#3585a

[Fukushima et al. 1994] M. Fukushima, Y. Oshima, and M. Takeda, Dirichlet forms and symmetric Markov processes, de Gruyter Studies in Mathematics 19, Walter de Gruyter, Berlin, 1994. Zbl 0838.31001

[Gefen et al. 1983] Y. Gefen, A. Aharony, and B. B. Mandelbrot, "Phase transitions on fractals. I. Quasilinear lattices. II. Sierpiński gaskets. III. Infinitely ramified lattices”, J. Phys. A 16:6 (1983), 1267-1278. 17 (1984), 435-444 and 1277-1289. MR 86g:82022a

[Hambly et al. 2006] B. M. Hambly, V. Metz, and A. Teplyaev, "Self-similar energies on postcritically finite self-similar fractals", J. London Math. Soc. (2) 74 (2006), 93-112. MR 2007i:31011 Zbl 1103.31005 
[Hansen and Zähle 2006] W. Hansen and M. Zähle, "Restricting isotropic $\alpha$-stable Lévy processes from $\mathbb{R}^{n}$ to fractal sets", Forum Math. 18:2 (2006), 171-191. MR 2007j:60124 Zbl 1106.60063

[Hohlfeld and Cohen 1999] R. G. Hohlfeld and N. Cohen, "Self-Similarity and the Geometric Requirements for Frequency Independence in Antennae", Fractals 7 (1999), 79-84.

[Hveberg 2005] K. Hveberg, Injective mapping systems and self-homeomorphic fractals, Ph.D. thesis, University of Oslo, 2005, Available at http://www.math.uio.no/ klara/thesis.pdf.

[Kigami 1993] J. Kigami, "Harmonic calculus on p.c.f. self-similar sets", Trans. Amer. Math. Soc. 335:2 (1993), 721-755. MR 93d:39008 Zbl 0773.31009

[Kigami 1994] J. Kigami, "Effective resistances for harmonic structures on p.c.f. self-similar sets", Math. Proc. Cambridge Philos. Soc. 115:2 (1994), 291-303. MR 95h:28012 Zbl 0803.60074

[Kigami 2001] J. Kigami, Analysis on fractals, Cambridge Tracts in Mathematics 143, Cambridge University Press, Cambridge, 2001. MR 2002c:28015 Zbl 0998.28004

[Kigami and Lapidus 1993] J. Kigami and M. L. Lapidus, "Weyl's problem for the spectral distribution of Laplacians on p.c.f. self-similar fractals", Comm. Math. Phys. 158:1 (1993), 93-125. MR 94m:58225 Zbl 0806.35130

[Koch and Denker 2005] S. Koch and M. Denker, "Hausdorff dimension for Martin metrics", pp. 163-170 in Algebraic and topological dynamics, edited by S. Kolyada et al., Contemp. Math. 385, Amer. Math. Soc., Providence, RI, 2005. MR 2007a:28007 Zbl 1102.28004

[Lindstrøm 1990] T. Lindstrøm, "Brownian motion on nested fractals", Mem. Amer. Math. Soc. 83:420 (1990), iv+128. MR 90k:60157 Zbl 0688.60065

[Metz 1993] V. Metz, "How many diffusions exist on the Vicsek snowflake?", Acta Appl. Math. 32:3 (1993), 227-241. MR 94m:31010 Zbl 0795.31011

[Metz 1996] V. Metz, "Renormalization contracts on nested fractals", J. Reine Angew. Math. 480 (1996), 161-175. MR 98b:31007 Zbl 0858.31008

[Metz 2003] V. Metz, "The cone of diffusions on finitely ramified fractals", Nonlinear Anal. 55:6 (2003), 723-738. MR 2005d:60129 Zbl 02005479

[Meyers et al. 2004] R. Meyers, R. S. Strichartz, and A. Teplyaev, "Dirichlet forms on the Sierpiński gasket”, Pacific J. Math. 217:1 (2004), 149-174. MR 2005k:31028 Zbl 1067.31011

[Mosco 2002] U. Mosco, "Energy functionals on certain fractal structures", J. Convex Anal. 9:2 (2002), 581-600. MR 2004a:28018 Zbl 1018.28005

[Musser 1999] G. Musser, “Wireless communications”, Scientific American (July 1999).

[Peirone 2006] R. Peirone, "Convergence of Dirichlet forms on fractals", pp. 139-188 in Topics on concentration phenomena and problems with multiple scales, vol. 2, edited by A. Braides and V. Chiadò Piat, Lect. Notes Unione Mat. Ital., Springer, Berlin, 2006. MR 2267882

[Puente-Baliarda et al. 1998] C. Puente-Baliarda, J. Romeu, R. Pous, and A. Cardama, "On the behavior of the Sierpinski multiband fractal antenna”, IEEE Trans. Antennas and Propagation 46:4 (1998), 517-524. MR 1615993 Zbl 0944.78529

[Rammal 1984] R. Rammal, "Spectrum of harmonic excitations on fractals", J. Physique 45 (1984), 191-206. MR 85d:82101

[Rammal and Toulouse 1983] R. Rammal and G. Toulouse, "Random walks on fractal structures and percolation clusters", J. Physique Letters 44 (1983), L13-L22.

[Sabot 1997] C. Sabot, "Existence and uniqueness of diffusions on finitely ramified self-similar fractals”, Ann. Sci. École Norm. Sup. (4) 30:5 (1997), 605-673. MR 98h:60118 Zbl 0924.60064

[Stanley et al. 2003] J. Stanley, R. S. Strichartz, and A. Teplyaev, "Energy partition on fractals", Indiana Univ. Math. J. 52:1 (2003), 133-156. MR 2004a:31006 Zbl 1037.31011 
[Stinchcombe 1989] R. B. Stinchcombe, "Fractals, phase transitions and criticality", Proc. Roy. Soc. London Ser. A 423:1864 (1989), 17-33. Fractals in the natural sciences. MR 91c:58084

[Strichartz 1999a] R. S. Strichartz, "Analysis on fractals", Notices Amer. Math. Soc. 46:10 (1999), 1199-1208. MR 2000i:58035

[Strichartz 1999b] R. S. Strichartz, "Isoperimetric estimates on Sierpinski gasket type fractals", Trans. Amer. Math. Soc. 351:5 (1999), 1705-1752. MR 99h:28021 Zbl 0917.28005

[Strichartz 2000] R. S. Strichartz, "Evaluating integrals using self-similarity", Amer. Math. Monthly 107:4 (2000), 316-326. MR 2001f:26011 Zbl 1009.26011

[Strichartz 2003] R. S. Strichartz, "Function spaces on fractals", J. Funct. Anal. 198:1 (2003), 4383. MR 2003m:46058 Zbl 1023.46034

[Strichartz 2006] R. S. Strichartz, Differential equations on fractals, Princeton University Press, Princeton, NJ, 2006. A tutorial. MR 2007f:35003

[Teplyaev 2004] A. Teplyaev, "Energy and Laplacian on the Sierpiński gasket", pp. 131-154 in Fractal geometry and applications: a jubilee of Benoît Mandelbrot. Part 1, edited by M. L. Lapidus and M. van Frankenhuijsen, Proc. Sympos. Pure Math. 72, Amer. Math. Soc., Providence, RI, 2004. MR 2006i:31011 Zbl 02154220

[Teplyaev $\geq 2007$ ] A. Teplyaev, "Harmonic coordinates on fractals with finitely ramified cell structure", Canadian Journal of Mathematics. To appear.

[Tyson and $\mathrm{Wu} 2005]$ J. T. Tyson and J.-M. Wu, "Characterizations of snowflake metric spaces", Ann. Acad. Sci. Fenn. Math. 30:2 (2005), 313-336. MR 2006g:54033 Zbl 02223085

[Tyson and $\mathrm{Wu}$ 2006] J. T. Tyson and J.-M. Wu, "Quasiconformal dimensions of self-similar fractals”, Rev. Mat. Iberoamericana 22:1 (2006), 205-258. MR 2268118 Zbl 1108.30015

[Zähle 2005] M. Zähle, "Harmonic calculus on fractals—a measure geometric approach. II", Trans. Amer. Math. Soc. 357:9 (2005), 3407-3423. MR 2005m:28027

Received August 18, 2006. Revised May 25, 2007.

\title{
BRIGHID BOYLE
}

brighidboyle@yahoo.com

\section{KRISTIN CEKALA}

kristin.cekala@prudential.com

DAVID FERRONE

David.Ferrone@uconn.edu

\section{NEIL RIFKIN}

Neil.Rifkin@uconn.edu

\author{
Alexander TeplyaeV \\ DEPARTMENT OF MATHEMATICS \\ UNIVERSITY OF CONNECTICUT \\ STORRS, CT 06269-3009 \\ UNITED STATES \\ teplyaev@math.uconn.edu \\ http://www.math.uconn.edu/ teplyaev
}

